modern government, this collection of essays well merits this more permanent form of presentation. They contain much to interest the scientist, although the place of the scientific or technical expert in government is discussed only in general terms by Mr. H. R. C. Greaves in his essay on the structure of the Civil Service.

R. BRIGHTMAN

\section{THE TABULATION OF MATHEMATICAL FUNCTIONS}

Tables of the Function arc $\sin z$

By the Staff of the Computation Laboratory. (Annals of the Computation Laboratory of Harvard University, Vol. 40.) Pp. xxxviii + 586. (Cambridge, Mass. : Harvard University Press ; London: Oxford University Press, 1956.) 100s. net.

Spheroidal Wave Functions including Tables of Separation Constants and Coefficients

By J. A. Stratton, P. M. Morse, L. J. Chu, J. D. C. Little and F. J. Corbató. (Technology Press Books in Science and Engineering.) Pp. xiii +613 . (Cambridge, Mass. : 'Iechnology Press of the Massachusetts Institute of 'Technology ; New York : John Wiley and Sons, Inc. ; London : Chapman and Hall, Ltd., 1956.) 100s, net.

7 HESE two books of tables illustrate an interesting contrast in the problems presented in tabulating a mathematical function, and in the methods of dealing with these problems. Both were computed on automatic computers, both come from Cambridge, Massachusetts, and both are substantial, but with this, resemblance practically ceases.

The first provides a definitive table of a standard mathematical function $f(z)=\sin ^{-1} z$ or arc $\sin z$ over the complex plane of $z=x+i y$. By symmetries of the function, only positive values of $x$ and $y$ need bo considered as tabular arguments. There is, however, a singularity at $z=1$. The tables give six-decimal values of real and imaginary parts of $f(z)$ for intervals in $x$ and $y$ which are small near $z=1$; in fact, $\delta x=\delta y=0.002$, while $x$ and $y$ extend over the intervals $0.916 \leqslant x \leqslant 1.090, \quad 0 \leqslant y \leqslant 0 \cdot 140$, re spectively. The intervals are successively increased to $\delta x=\delta y=5$, for which values the total extent of the table is $0 \leqslant x \leqslant 195$ with $125 \leqslant y \leqslant 475$, and $200 \leqslant x \leqslant 475$ with $0 \leqslant y \leqslant 350$. To aid interpolation, real and imaginary parts of $h \mathrm{~d} f / \mathrm{d} z$ and $\frac{1}{2} h^{2} \mathrm{~d}^{2} f / \mathrm{d}^{2}$ are tabulated; in this $h=\delta x=\delta y$ is the tabular interval. The unorthodox use of $\delta$ for $h$ in table headings is misleading and requires a word of warning; for example, $\delta f^{\prime}(z)$ does not mean a difference, but $\delta \times \mathrm{d} f(z) / \mathrm{d} z$. The presentation and printing of the tabular material are otherwise excellent. An introduction describes the properties of the function $\sin ^{-1} z$, and the details of the calculation and checking, and of interpolation into the tables. The writer has not tested the tables for accuracy, but the care taken by the Harvard Computation Laboratory and its experience in making mathematical tables are well known, and accuracy can be expected to be high, probably perfect. The tables were prepared on the Harvard Mark IV Calculator.

The second table should, perhaps, be regarded as, in a sense, experimental-though a highly developed and substantial experiment. The spheroidal wave. functions tabulated involve several parameters and depend on theory of relatively recent development. The former consideration rules out anything like a really complete tabulation (though an even more substantial volume of actual solutions is foreshadowed), and the latter may mean that the best form of tabulation has not yet been found. This appears to be true even of Mathieu functions, which involve a parameter fewer. The authors must be congratulated on a really substantial attempt to make the spheroidal wave-functions readily available to workers in this field. Two sets of tables are given-for prolate and oblate spheroidal wavefunctions, respectively. The wave-functions themselves are not tabulated, but are given in the form of expansions in series of spherical Bessel functions $j_{n}+m(\xi)=(\pi / 2 \xi)^{\frac{1}{2}} J_{n+m+1 / 2}(\xi)$ in the case of radial functions $J$, which satisfy the differential equation that arises from Laplace's equation by separation of variables after transformation to spheroidal coordinates, namely,

$$
\frac{\mathrm{d}}{\mathrm{d} \xi}\left[\left(\xi^{2}-1\right) \frac{\mathrm{d} J}{\mathrm{~d} \xi}\right]-\left(A-h^{2} \xi^{2}+\frac{m^{2}}{\xi^{2}-1}\right) J=0
$$

or

$$
\frac{\mathrm{d}}{\mathrm{d} \xi}\left[\left(\xi^{2}+1\right) \frac{\mathrm{d} J}{\mathrm{~d} \xi}\right]-\left(A-g^{2} \xi^{2}-\frac{m^{2}}{\xi^{2}+1}\right) J=0
$$

(oblate)

or in series of associated Legendre functions $P_{m+n}^{m}(\eta)$ in the case of angular functions $S$, which satisfy the differential equation

$$
\frac{\mathrm{d}}{\mathrm{d} \eta}\left[\left(1-\eta^{2}\right) \frac{\mathrm{d} S}{\mathrm{~d} \eta}\right]+\left(A^{2}-h^{2} \eta^{2}-\frac{m^{2}}{1-\eta^{2}}\right) S=0
$$

or

$$
\frac{\mathrm{d}}{\mathrm{d} \eta_{i}}\left[\left(1+\eta^{2}\right) \frac{\mathrm{d} S}{\mathrm{~d} \eta}\right]-\left(A^{2}-g^{2} \eta^{2}-\frac{m^{2}}{1-\eta^{2}}\right) S=0
$$

(oblate)

In these, $m$ (an integer) and $A$ are 'separation constants'. The tables give coefficients in all four expansions for $m=0(1) 8, l=m(1) 8$, and with $h$ or $g=0(0 \cdot 1) 1(0 \cdot 2) 8$. Seven-figure values are given for coefficients down to about $10^{-10}$ for radial functions, and down to about $10^{-18}$ or $10^{-20}$ for some angular functions. The separation-constants $A$ are not given directly, but can be obtained from sevenfigure values of $t$ where

$$
A=l(l+1)+h^{2}\left[\frac{2 l(l+1)-2 m^{2}-1}{(2 l-1)(2 l+3)}+t\right]
$$

The tables were produced on the Massachusetts Institute of Technology's computer, Whirlwind I, and have been reproduced quite well, though both format (with its too lavish use of space) and typed material reproduced are inferior to those of the Harvard volume; they are nevertheless adequate.

The introductory matter consists of a reprint of an article by Chu and Stratton from J. Math. Phys., 20, 259-309 (1941), and a rather too sketchy introduction to the tables by Little and Corbato. This is a valuable pioneer table.
J. C. P. MrLler 